\title{
LOS DISTRITOS INDUSTRIALES ANTE EL RETO DE LA GLOBALIZACIÓN
}

\author{
Fabio Sforzi \\ Facultad de Economía, Universidad de Turín (Italia)
}

\section{Introducción}

El reto de la globalización se juega en el terreno de la defensa dinámica de la diversidad cultural. Para poder concretar una integración económica mundial progresiva, con las ventajas que ello comporta tanto para las economías desarrolladas como para las que están en vías de desarrollo, que lleve a una disminución de la desigualdad de las condiciones de vida, no es necesario reducir la diversidad cultural hasta convertir las culturas locales, tan distintas entre sí, en culturas uniformes.

La uniformidad de los numerosos sistemas locales que constituyen la economía mundial es una condición socio-cultural que beneficia a las estrategias productivas y de comercialización de las empresas transnacionales. Éstas se basan en un sistema de producción de masa, y se organizan como empresa-red a través de redes globales. La economía mundial entendida como un sistema de redes globales, carente de identidades territoriales, representa la idea de globalización que posee la mayor parte de las empresas transnacionales. La indiferencia hacia los lugares de producción y de vida es una actitud común en gran parte de las organizaciones empresariales de los países industrializados, tanto de vieja como de reciente industrialización, y en las organizaciones mundiales más influyentes, como el Fondo Monetario Internacional y la Organización Mundial de Comercio.

Si bien es cierto que las empresas transnacionales son políticamente poderosas - llegan a condicionar las estrategias económicas de los estados nacionales - y económicamente importantes - 500 empresas transnacionales controlan el $70 \%$ del comercio mundial y el $1 \%$ es responsable de la mitad de las inversiones directas extranjeras del mundo (Hines, 2000) - también es verdad que éstas no representan la totalidad de las empresas mundiales ni tampoco son el único modelo con éxito.

Existen economías nacionales - pertenecientes al Grupo de los 8 países más industrializados del mundo, como Italia - que deben su dinamismo al hecho de haber 
organizado la producción de manera completamente distinta, según el modelo del distrito industrial, concretamente a través de sistemas locales de pequeña y mediana empresa. Se trata, como es bien sabido, de una fórmula empresarial para la que son muy ventajosos el arraigo territorial de las empresas y la variedad de las necesidades percibidas por grupos de consumidores distribuidos en los distintos sistemas locales del mundo, necesidades que dichas empresas son capaces de satisfacer a través de un sistema de producción en pequeña serie, que permite la elaboración de productos por encargo de los clientes. La economía mundial, entendida como un calidoscopio - es decir, una variedad mutable - de sistemas locales, dotado cada uno de ellos de su propia peculiaridad cultural y económica, explícita o potencial (en este caso, que ayude a manifestarse) y con múltiples interdependencias, gracias a los tipos de intercambios, mercantiles o no, que se realizan entre ellos, representa la idea de globlización que está detrás del modelo del distrito.

Por ello, existen al menos dos formas de concebir y orientar la globalización, o de gobernarla, al no poderse comparar el poder de las empresas transnacionales con el de los distritos industriales en cuanto al grado de influencia de los gobiernos nacionales.

En el fondo se trata del enfrentamiento entre dos tendencias. No puede hablarse todavía de dos políticas económicas, ya que de momento existe sólo una dominada por los intereses de las empresas transnacionales, pero podría llegar a hacerse realidad. Desde el enfoque de los distritos industriales, un proceso de globalización cuyo avance se debiera al empuje incontestable de las empresas transnacionales reduciría la variedad de las culturas locales y supondría un peligro para sus perspectivas de desarrollo. Por otra parte, los distritos industriales constituyen un caso concreto - no simplemente un modelo teórico - de una manera de producir que exige al mismo tiempo arraigo local y apertura global. Por su capacidad para integrar dinámicamente estas dos dimensiones interdependientes del modo de actuar económico contemporáneo, los distritos han demostrado que pueden lograr el éxito económico (para las empresas) y garantizar prosperidad (para la sociedad local). Por tanto, se puede imaginar una política económica multicultural de alcance mundial que haga hincapié en la diversidad cultural reflejada en la variedad de las realidades locales y en la posibilidad de estas últimas de constituir otros tantos sujetos económicos - allí donde fuera posible, y en general en cualquier parte del mundo donde se den las potencialidades - para poner en marcha procesos de desarrollo local.

\section{Acerca de la globalización}

\subsection{Una clave de lectura}

Para desarrollar el argumento de este informe conviene definir previamente qué se entiende por globalización y orientar luego el razonamiento en ese sentido. 
Entre las distintas definiciones de que disponemos, la que nos parece más convincente es la que afirma que globalización es el "término [...] con el que se manifiesta la tendencia de las empresas a desarrollar conjuntamente procesos de internacionalización y de informatización" (EEG, 1995, p. 526). La internacionalización indica el proceso de apertura hacia el exterior por parte de las empresas, que se realiza a través de a) el comercio internacional, b) la inversión directa productiva y c) la financiera - según algunos, los movimientos internacionales de capital son la manifestación más evidente del proceso de globalización. Estas tres formas de internacionalización se ven favorecidas por las privatizaciones y las liberalizaciones realizadas por numerosos estados nacionales. Todo esto reduce las barreras a los intercambios comerciales y a las inversiones, y provoca un aumento de la competencia; sin embargo reduce también el control democrático de los estados y de sus respectivas sociedades nacionales sobre la propia economía.

Se trata de procesos que, en contacto con las tecnologías de información y de comunicaciones, reciben de éstas un empuje y una aceleración. El desarrollo de las tecnologías de información y de comunicaciones está relacionado muy estrechamente con la globalización, y representa, de hecho, uno de los elementos motores.

Si bien las tecnologías de información y de comunicaciones facilitan sobre todo los movimientos de capital - siendo factible trasladar capitales de un país a otro en tiempo real con los mercados financieros que operan las 24 horas del día - no son, sin embargo, menos importantes para la descomposición internacional del proceso productivo, desde el momento que permiten a una empresa, gracias a un sistema informativo centralizado, dirigir en tiempo real cada una de las fases de producción, cuyo desarrollo tiene lugar en los establecimientos que ésta posee distribuidos por todo el mundo (véase el caso de la Fiat en Italia). La reorganización de la gran empresa fordista en empresa-red a escala mundial ha sido el factor principal de empuje del proceso de globalización.

La empresa-red, que sigue contemplándose en la producción de masa, aunque "diferenciada" de lo que era para la tradición fordista, adopta una estrategia de localización y de división del trabajo pluriterritorial a escala mundial.

A diferencia de la gran empresa de matriz fordista - que operaba según un proceso verticalmente integrado - la empresa-red se constituye a través de unidades productivas diferentes en partes de producto, fases de elaboración o servicios a la producción y de comercialización. A través de redes logísticas dirigidas por empresas especializadas, se transportan los productos intermedios al establecimiento en el que se realiza el producto final, mientras las informaciones concernientes a las relaciones operativas cotidianas viajan por las redes telemáticas. 


\subsection{Los orígenes}

El fracaso del sistema de Bretton Woods (1971) suele ser considerado comúnmente el punto de arranque del proceso de globalización. Asimismo, los primeros años setenta marcaron el principio de la crisis del fordismo, por efecto de la crisis de petróleo (1973) y de la saturación de los mercados de masa. Ante la crisis, las grandes empresas reaccionaron, sobre todo las americanas, aprovechándose del "desorden" que siguió al final del sistema de Brettn Woods, y utilizando la libertad de movimiento de capitales, coordinada a través de los mercados en vez de regulada por una autoridad central controlada políticamente. Este hecho permitió la puesta en marcha de procesos de desindustrialización por parte de las grandes empresas, que llevaron al extranjero parte de la producción y así recuperaron los precios competitivos, buscando en la investigación tecnológica soluciones que hicieran más fácil esta estrategia de reorganización productiva. La revolución micro-electrónica así como la aparición de sistemas manufactureros flexibles que transformaron la forma de producir forman parte de esta estrategia.

Se puede estar de acuerdo en mayor o menor medida sobre el papel propulsor que tuvieron las grandes empresas en la puesta en marcha del proceso de globalización, pero lo que sí es cierto es que, a estas alturas, la globalización se expande bajo su guía. Prevalecen las exigencias del mercado de las empresas transnacionales, tanto desde el punto de vista de la producción como de la comercialización, a la hora de definir las reglas de gobernabilidad de la economía mundial.

\subsection{El «nuevo» americanismo}

Para poder consolidar un modelo productivo que perdure, éste ha de integrarse en la misma sociedad local en que se desarrolla el proceso de producción, generando valores, comportamientos típicos e instituciones acordes con las exigencias de la organización productiva en la que se basa dicho modelo productivo.

Con esta declaración se pretende poner en evidencia la conexión, entre economía y sociedad, que ha encontrado la experiencia de los distritos y que ha despertado el interés de los estudiosos y de los responsables políticos. La afirmación de que el distrito industrial es una máquina social antes que una fórmula productiva pretende justamente recordar que la forma de organizar la producción - ya sea fordista o de distrito - no puede obviar la forma en que está organizada la sociedad. Hay una interrelación entre ambos componentes de modo que se considera que el cambio económico es también social y viceversa. El éxito o fracaso de cualquier sistema socio-económico local depende del modo en que se interprete y se reproduzca dicha transformación en el tiempo. 
Si gracias al distrito industrial se ha vuelto a descubrir ese nexo entre economía y sociedad que explica la consolidación de un modelo productivo y su cambio, en el modelo de producción fordista-taylorista sin embargo ese nexo tenía un valor normativo, como ya subrayaba Gramsci (1975) en "Americanismo y fordismo":

"El nuevo método de trabajo y la manera de vivir son indisolubles: no se pueden obtener éxitos en un campo sin obtener, al mismo tiempo, resultados tangibles en el otro. En América, la racionalización y el prohibicionismo están sin lugar a dudas relacionados, y las encuestas de los industriales sobre la vida de los obreros, o el servicio de inspección, creado por algunos industriales para controlar la "moralidad" de los obreros, son exigencias del nuevo método de trabajo. Quienes se burlasen de estas iniciativas o viesen en ellas tan solo una manifestación hipócrita de "puritanismo" se negarían toda posibilidad de comprender la importancia, el significado y el alcance objetivo del fenómeno americano, que supone además el mayor esfuerzo colectivo [realizado hasta ahora], para crear con una rapidez extraordinaria y con una clarividencia del fin perseguido nunca vista en la historia, un tipo nuevo de trabajador y de hombre" (p. 489).

Naturalmente las modalidades de la interrelación socio-económica local varían con la transformación que va sufriendo el modelo productivo que define esa realidad. Mientras en el modelo fordista es la sociedad la que debe adaptarse a las exigencias del método productivo, y por tanto resulta subordinada a las exigencias de la empresa, en el modelo del distrito, en cambio, ocurre exactamente lo contrario, ya que es la sociedad local la que orienta la producción, y ésta está influida por las dinámicas sociales, tanto en sentido positivo como negativo. De este modo, los beneficios o las satisfacciones, morales y materiales, que una sociedad local recibe de "su" industria difieren, dependiendo de cómo dicha sociedad esté involucrada en la producción.

Sigamos leyendo a Gramsci: "El Taylor traduce con cinismo y sin sobreentendidos el fin de la sociedad americana: desarrollar en el hombre trabajador al máximo la parte maquinal y romper el viejo nexo psico-físico del trabajo profesional calificado, que pedía la participación de la inteligencia, de la iniciativa y de la fantasía del trabajador, para reducir únicamente a su aspecto físico las operaciones de producción" (p. 489).

Justamente ese tipo de participación colectiva en el trabajo constituye la base de los modernos distritos industriales y ha permitido su reproducibilidad en los años pasados.

"Desde este punto de vista hay que ver las iniciativas "puritanas" de los industriales tipo Ford. Es evidente que ellos no se preocupan de la "humanidad" ni de la "espiritualidad. del trabajador, que quedan aplastadas. Esta humanidad y espiritualidad estaban protegidas, en cambio, en el mundo del trabajo de la "creación" productiva: 
máxima en el ámbito de la artesanía, donde la individualidad del trabajador quedaba plasmada en el objeto creado, donde se fundían arte y trabajo. Pero es justamente contra esta forma de humanidad y de espiritualidad contra la que lucha el nuevo industrialismo. Las iniciativas "puritanas" tienen sólo este fin: mantener un equilibrio psico-físico fuera del trabajo, para impedir que el nuevo método lleve al colapso fisiológico del trabajador. Este equilibrio es puramente exterior, por ahora no es interior. El equilibrio interior no puede ser creado más que por el mismo trabajador y por su sociedad, con medios propios y originales" (p. 490).

La expresión "mundo de la producción creativa" consigue una buena definición del modelo del distrito respecto al modelo fordista-taylorista, que le exige al trabajador sólo un esfuerzo físico, lo que lo convierte en una cantidad constante, considerando el trabajo como una simple mercancía y negándole de esta manera al trabajador la posibilidad de cambiar con el aprendizaje a través de la experiencia, individual y colectiva. Por otra parte, a un "gorila amaestrado" (según la conocida expresión de Taylor) se le pide sólo que ejecute, no que tome iniciativas en el curso del proceso productivo, y mucho menos que tome parte de forma consciente. Lo que cuenta es la productividad del trabajo, no la capacidad innovadora del trabajador.

Para seguir usando las palabras de Gramsci, al fordismo (modelo de producción de masa) le ha hecho falta el americanismo (modelo de sociedad de masa) para poder realizarse y afianzarse en Estados Unidos como modelo nacional de industrialización y, a continuación, difundirse en los demás países capitalistas desarrollados, pese a las inevitables variantes.

Análogamente, la globalización entendida como modelo de producción de masa transnacional necesita, para su realización y consolidación en el mundo, un "nuevo" americanismo, o sea, un modelo de sociedad de masa a escala mundial: por lo que se refiere a la producción, encontrando países dispuestos a albergar instalaciones productivas de empresas transnacionales, en detrimento de la formación de una propia industria nacional; en lo referente al consumo, creando un mercado global donde los gustos de los consumidores estén nivelados, bajo la influencia del modelo cultural americano, que impulsa al consumo de productos globales de masa.

\section{El reto de la globalización}

\subsection{De la producción al consumo}

La literatura sobre el cambio económico global hace hincapié en el reto que la globalización ha supuesto para los distritos industriales, centrándose sobre todo en 
las transformaciones sufridas en la organización de la producción. El desarraigo del proceso de producción, respecto a su entorno primitivo, unido a la reorganización internacional del proceso productivo y a la desterritorialidad del factor principal de la producción, que es el conocimiento, - ahora accesible on-line y no a través de la pertenencia a un territorio, pues la fluidez de las redes virtuales garantiza su circulación sin el entorpecimiento que suponen las costumbres sociales sedimentadas en dicho entorno - han capturado el interés de la mayoría de estudiosos.

Quien escribe considera que esta reflexión no capta, sin embargo, algo esencial: el nexo existente entre producción y consumo. Si no se comprende la importancia que el consumo tiene para la actividad económica de producción, resulta difícil hasta sugerir el modo en que los distritos industriales deberían aprovecharse de la globalización, que no es simplemente un conjunto de elementos negativos. De esta convicción nace precisamente el acercamiento al tema que aquí se presenta del reto que implica la globalización para los distritos.

El reto que la globalización lleva hasta los distritos no tiene que ver con el modelo de producción, es decir, con su modo de producción, que de todas formas se benefícia con el cambio tecnológico, ni tampoco con la serie de bienes producidos sino con el modelo de consumo, o sea, con los efectos de homologación cultural que la globalización determina.

Esta tendencia a la homologación cultural se apoya en las estrategias de las empresas transnacionales que, si bien se diferencian de las grandes empresas de matriz fordista en el modo de organizar la producción (como ya se ha dicho anteriormente), siguen siendo concebidas, sin embargo, para la producción de masa, y por consiguiente, precisan todavía de una sociedad de masa para vender sus productos.

Por lo que se refiere a estos productos, las empresas transnacionales no representan una competencia directa para los distritos, ya que aquéllas intervienen en campos de consumidores diferentes: mercados uniformes (capaces de absorber grandes cantidades de la misma mercancía), mientras los distritos intervienen en mercados diferenciados (destinados a absorber cantidades limitadas de una misma mercancía). Sus productos son distintos porque se dirigen a una clientela distinta, distinto es también su sistema productivo - de pequeña escala y no de gran escala - distinta es su organización de la producción - redes de empresas emplazadas en el mismo territorio que cooperan, en lugar de una red pluri-territorial de unidades productivas, jerárquicamente dependientes o semi-independientes; descomposición local y no internacional del proceso productivo; economías externas de producción locales /territoriales y no internas de variedad/de empresa - y distinta es asimismo su estrategia de comercialización (oferta de proyectos de productos y, por tanto, una producción por encargo de la clientela, en lugar de la venta de productos ya fabricados a una clientela a la que hay que convencer de su adquisición). 
Las empresas transnacionales se vuelven competidores directos de los distritos desde el punto de vista del consumo. El éxito de las estrategias industriales depende de la expansión de un mercado mundial de consumidores, tendente a la uniformidad, donde poder vender a todo el mundo los mismos (o casi) productos. La persistencia de la diversidad cultural, nacional y local, el aumento de consumidores conscientes, con criterios propios incluso ante sus necesidades ordinarias, respaldados por su propia identidad cultural, constituyen el verdadero obstáculo para su consolidación definitiva a escala mundial.

Mientras para prosperar y reproducirse a los distritos les sirve un mundo culturalmente diversificado, a las empresas transnacionales, en cambio, para expandirse les sirve un mundo culturalmente homologado. Ambas tendencias están presentes dentro de la globalización y podrían convivir dentro de su marco, aunque el mantenimiento de la diversidad cultural disminuye las posibilidades de expansión mundial de las empresas transnacionales, lo que constituye un freno a su crecimiento.

En definitiva, el auténtico reto de la globalización en los distritos industriales deriva del intento de imponer en todo el mundo una monocultura de consumo.

\subsection{La importancia del territorio}

El problema para los distritos no es producir cada vez más, ni a bajo coste, sino cada vez mejor. Por eso, es indispensable la relación entre las empresas y la sociedad local, ya que esta última representa el ambiente cultural - que se mantiene unido gracias a un sistema de valores compartido - en el que se educa la mentalidad a un sistema económico-cooperativo, se generan las motivaciones que llevan a la actividad empresarial, en el que se crea la costumbre de pensar en productos siempre nuevos así como en las técnicas para realizarlos, en el que se forman las capacidades profesionales y organizativas, en el que se generan continuamente nuevas iniciativas empresariales, se proyectan misiones comerciales para explorar nuevos mercados en busca de clientes, para poder comprender cuáles son sus exigencias y ofrecerles, para satisfacerlas, la propia capacidad productiva.

Por todo ello, el distrito está "obligado" a una incesante actividad innovadora si quiere mantenerse en la frontera del cambio, lo que hace indispensable disponer de una eficiente red de canales de comunicación, formales e informales, que faciliten y alimenten la circulación de ideas y conocimientos en su interior, entre las mismas empresas, entre éstas y la sociedad local, y también con el resto del mundo. Las buenas ideas no surgen sólo de la cooperación entre las empresas y las personas que 
participan en el proceso productivo, sino también de las personas que tratan de entrar a formar parte del mismo, como empleados y nuevos empresarios; de forma distinta surgen igualmente de las relaciones operativas con los clientes, que son parte integrante del proceso productivo. Por último, nacen también del contacto con los ambientes creativos y de la innovación técnica, asî como de la comparación que se establece con los productos de la competencia. Los contactos comerciales con los posibles clientes se producen de las formas más insólitas, y menos planificadas, muy a menudo fuera de los circuitos institucionales (asociaciones de categoría, cámara de comercio, muestras especializadas etc.)

Los procesos principales que tienen lugar como consecuencia de la compenetración entre el aparato productivo y la sociedad local se pueden resumir con las palabras de Becattini (2000) en los siguientes apartados:

- la subdivisión progresiva, finalizada y autocontenida, de un proceso productivo principal y de otros complementarios e instrumentales;

- la creación y reproducción a lo largo del tiempo de nexos dinámicos entre capacidades productivas especializadas locales y núcleos de necesidades observadas en el mercado mundial;

- la sedimentación en instituciones - formales e informales, materiales e inmateriales - de praxis sociales que respetan simultáneamente las condiciones de la competencia y de reproducibilidad social y ecológica local;

- la integración dinámica entre saber productivo contextual, a menudo tácito, y saber productivo científico-técnico, o codificado;

- la formación de figuras profesionales y organizaciones (integradores versátiles) que median entre la exigencia de especialización y la de versatilidad;

- la formación, la consolidación y la transformación del sentimiento de pertenencia, lealtad y confianza en los agentes humanos de la producción;

- la alimentación constante de la movilidad social y profesional a través del mercado de trabajo y de la incubación empresarial.

La salvaguardia de los procesos que constituyen la base de la reproducción social del distrito es la tarea principal de las organizaciones de los distritos industriales, representantes de intereses generales y sectoriales, a través del proyecto y realización de políticas apropiadas que puedan hacer frente a las presiones que sufre desde el 
exterior la sociedad local. La sociedad del distrito industrial, al igual que las demás sociedades locales, no es inmune a los procesos de homologación cultural que lleva consigo la globalización y que pueden amenazar la reproducibilidad más que los contrastes que surgen en el interior.

Durante los años en que triunfó el fordismo, llegar a ser consciente de representar un modelo económico y social que podía garantizar prosperidad a sus protagonistas fue tarea ardua y difícil, y los agentes económicos del distrito, junto a la entera sociedad local, tuvieron que superar los obstáculos ideológicos de los defensores del modelo de industrialización americano. En la era de la globalización, una vez más "la historia se repite". La capacidad de persuasión de los predicadores de las redes globales como frontera de la modernidad económica y social, podría hacer creer a algunos agentes del distrito "que crean opinión" que pueden prescindir de la sociedad local - un lastre que hay que arrastrar por el sendero del desarrollo, que no permite crecimientos económicos individuales vertiginosos, sino un crecimiento económico colectivo moderado, que asegura una alta cohesión social, fuerza y debilidad del modelo del distrito, pero sobre todo, una garantía de continuidad en el tiempo - e imprimir una aceleración al crecimiento económico sin preocuparse de aflojar las relaciones sociales sustituyéndolas con relaciones virtuales, porque los "contextos telemáticos" son más simples de manejar que los contextos territoriales. Por otra parte, hay quienes sostienen que en los contextos telemáticos intervienen "los mismos factores que influyeron en la capacidad de los distritos de constituir a lo largo del tiempo una infraestructura eficaz comunicativa y cognoscitiva" (Corò y Micelli, 1999, p. 25), subestimando el papel insustituible de integrador versátil desempeñado por la sociedad local, o lo que es lo mismo por el territorio.

Por eso, la desterritorialidad de la producción, que imita las estrategias de industrialización de las empresas transnacionales, si bien es aconsejable para empresas cuyas economías de producción son virtualmente indiferentes al arraigo local, resulta en cambio un contrasentido para las empresas del distrito. Pensar en sustituir la sociedad local por una sociedad virtual, con la perspectiva de reproducir las ventajas del territorio eliminando las relaciones sociales significa no haber comprendido la lección del distrito industrial.

Con estas consideraciones no se les pretende negar a los distritos la posibilidad de aprovechar las oportunidades que les ofrecen las nuevas tecnologías de información y de comunicación - y de la "nueva economía" en general - para modernizar la organización productiva. Sin embargo, el objetivo no puede ser el de desarraigar la producción de los lugares en los que esta tiene lugar, en simbiosis con la sociedad local, sino el de incrementar la eficacia de las redes inter-locales, de colaboración entre empresas pertenecientes a diferentes sistemas locales, que actualmente existen ya y son numerosos, y extenderlos a otros lugares productivos. La participación en una red virtual no supone necesariamente un cambio radical de los procesos productivos, y permite un uso táctico del medio informático. 


\subsection{Las posibles respuestas}

Llevar el reto de la globalización al terreno del consumo significa trabajar en la actualización de la forma de relacionar la producción local con el mercado mundial, apostando por el papel principal de las estructuras de organización comercial.

Significa aprovechar al máximo las oportunidades que la globalización pone a nuestra disposición para contraponer a la homologación de los consumos la diferenciación de los consumos, valorizando de esta manera la diversidad cultural, contra la nivelación y la imposición de la nueva one best way cultural que lleva consigo la globalización incuestionable de las empresas transnacionales. Una estrategia basada en la diferenciación de las culturas locales, considerándola un factor competitivo ventajoso, coherente con la lección del distrito, que se apoya en las redes virtuales globales y en las redes logísticas. Por otra parte, los flujos de ideas, informaciones y tecnología que circulan por las redes globales pueden orientarse hacia estrategias que potencien y favorezcan el desarrollo de economías locales en el mundo.

La disponibilidad de tecnologías de información y comunicación permite utilizar las redes virtuales globales como un vehículo a través del que difundir informaciones sobre la adquisición, que den a conocer la variedad de productos especializados, capaces de satisfacer núcleos de necesidades corrientes, además de especiales, como alternativa a los productos de masa. De este modo, se puede educar a los consumidores a una alternativa y a una elección, apartándolos de la vía del conformismo y estimulando en ellos el deseo de variedad y diferenciación.

Se trata de dotar a los distritos industriales de estrategias globales de comunicación y de imagen, como instrumento de organización comercial que se concreta en el comercio electrónico, como sistema de venta y de penetración en el mercado mundial, acompañando y completando los canales tradicionales de intermediación mercantil, para mejorar el servicio que se les ofrece a los clientes - haciéndole frente a la competencia - y para hacerse con clientes nuevos.

En los distritos industriales, la intermediación mercantil es una actividad realizada por las empresas de servicios (individuales o con empleados) que cooperan con las empresas manufactureras y que tienen como objetivo lograr la conexión entre éstas y la clientela mundial. Se trata de agentes económicos que exploran los distritos industriales, por encargo de los clientes, en busca de empresas que posean la experiencia técnica necesaria para realizar, previo pedido del cliente, productos cuyas características específicas (originalidad, estética, calidad de los materiales y de la elaboración, precio) respondan a las exigencias concretas de dicho cliente. Su función consiste en seleccionar las empresas productoras, convencer al cliente de que el producto que necesita puede ser fabricado por estas empresas, colaborar con las empresas y con los clientes en la realización de proyectos de producto que pongan en práctica la idea inicial. 
Por todo esto, es fundamental que el intermediario tenga conocimientos técnicos de los productos, que conozca su proceso de fabricación y que sepa también cuáles son los recursos productivos de la empresa para poder estimar su idoneidad o no a la hora de realizar el producto.

El cumplimiento de esta función exige además un buen conocimiento de cuál es la variedad de la oferta local - en cuanto a las empresas productoras, abastecedoras de material etc. - así como cuáles son las expectativas del cliente. Las relaciones intermediario/cliente e intermediario/empresa se construyen teniendo como bases la confianza y la capacidad profesional.

Las páginas web de intermediación on-line no hacen de estas capacidades profesionales algo obsoleto, más bien al contrario, pues ellas se convierten en una condición necesaria para la consolidación de nuevas formas de organización comercial, utilizando todas las posibilidades que las redes virtuales les ofrecen.

La imagen de un producto fabricado en el distrito no requiere la manipulación de una hábil campaña publicitaria, donde se inventan contextos virtuales para promocionar el producto, pues ésta halla una correspondencia tangible en el sustrato cultural local o nacional, del que el distrito forma parte, y en una tradición que, lo mismo que la innovación, se incorpora al producto que el distrito ha realizado, convirtiéndose en un rasgo suyo concreto.

Capacidad de proyectar, experiencias técnicas de fabricación, modernidad y tradición, identidad y calidad de los productos son características sobre las que también se puede hacer hincapié a través de un sitio de intermediación on-line, dándoles al cliente-empresa o al consumidor final la posibilidad de hacer frente a la uniformidad cultural de la producción de masa, comparando los productos específicos con los productos estándar, y constatando que por el mismo precio existe gran posibilidad de elección dentro de una amplia gama de productos distintos que se pueden pedir o adquirir directamente, y que están además a su alcance.

La expansión del consumo de productos especializados, en la medida en que valoriza la diversidad cultural, trabaja a favor de la producción de los distritos (bienes para el consumo personal y del hogar, que incluyen también los bienes instrumentales que se necesitan para producirlos, y productos agro-alimenticios), pero les concede asimismo la oportunidad a los productos típicos de las distintas realidades locales, que se hallan fuera del proceso de producción de masa y de los circuitos comerciales tradicionales, de que entren en el mercado mundial.

La actualización de los dispositivos de intermediación mercantil a través de las redes virtuales globales se completa con la utilización de redes logísticas especializadas que suponen un apoyo real para las transacciones comerciales efectuadas en el ámbito del mundo virtual. La entrega "efectiva" de las mercancías una vez que las han 
pedido las empresas-clientes o los consumidores finales, en el plazo de tiempo acordado, representa la condición necesaria de factibilidad y de resultado para el desarrollo progresivo del comercio electrónico.

Una estrategia eficaz, como respuesta al reto que supone la globalización, les exige a los distintos gobiernos nacionales un papel activo dentro de las organizaciones internacionales donde se toman las decisiones políticas, relativas a la normativa que regula la fabricación de los productos especializados (por ejemplo, la Unión europea) y su comercialización (por ejemplo, la Organización Mundial de Comercio). Y no se trata de proteger estos productos sino de salvaguardar su peculiaridad, su originalidad en la técnica de fabricación, así como la fórmula organizativa en la que se apoyan, en cuanto resultado de una tradición cultural que está en contra de las prioridades de las empresas transnacionales que empujan hacia una homologación productiva de masa.

Los gobiernos regionales deben intervenir también en este proceso, pues la exigencia de atraer inversiones productivas extranjeras, para reindustrializar un territorio o sacarlo de la depresión económica en que se encuentra, hace olvidar a menudo la importancia de sus peculiaridades culturales y de sus potencialidades endógenas. Se prefiere la creación inmediata de puestos de trabajo, indiferentes a su duración en el tiempo o a los efectos que esto puede acarrear a la hora de mejorar las competencias laborales de los trabajadores empleados, en lugar de promocionar la formación de una industria local en la que intervengan también inversiones extranjeras. Estando así las cosas se dispersan los conocimientos, las competencias profesionales y experiencias productivas que, oportunamente sostenidos y actualizados bajo el perfil de la organización productiva y comercial, podrían crear un ambiente que incentivase el espíritu de iniciativa y empresario, enfocado a la producción de bienes específicos para colocar dentro del mercado mundial.

\section{Algunas consideraciones finales}

En este informe que nos ocupa se propone una lectura sobre el reto que lleva la globalización a los distritos industriales radicalmente distinta del punto de vista imperante sobre este tema, según el cual el modelo de los distritos perderá competitividad si no es capaz de imitar a las empresas transnacionales en sus estrategias de reorganización internacional de la producción, aprovechando las oportunidades que proporcionan las tecnologías de información y de comunicación. Lo que en este informe se sostiene, sin embargo, es que el modelo de los distritos debe beneficiarse de esta oportunidad desde el consumo y no desde la producción, adoptando estrategias de comercialización innovadoras que tienen lugar, por ejemplo, con el respaldo del comercio electrónico en cuanto instrumento de intermediación mercantil. 
Por otra parte, no hay más que leer la vulgata sobre "nueva economía" para darse cuenta de que los principios organizativos y humanos en los que ella se basa son muy parecidos a los del modelo de los distritos: versatilidad, innovación, capacidad empresarial, talento creativo, valorización del capital humano en el proceso productivo, relaciones de cooperación entre las empresas, servicio de asesoría al cliente, programas de producción basados en los pedidos, relaciones muy estrechas y continuadas entre empresas y clientes etc.

Existe desde luego dentro del distrito industrial la necesidad de desarrollar iniciativas productivas y no sólo de comercialización, que utilicen las posibilidades de las redes virtuales globales sobre todo, para hacer mucho más eficaces los intercambios mercantiles inter-locales, pero sin desestabilizar el contexto territorial del que dependen los factores de su reproducibilidad social. Éste es el vínculo de sostenimiento social al que debe ajustarse cualquier innovación tecnológica que utilice la red para una parte del proceso productivo del distrito.

Las amenazas de la globalización para los distritos industriales se localizan principalmente en la reducción de la diversidad cultural, a escala mundial, que genera la globalización bajo el impulso de las empresas transnacionales, funcional para la expansión de estas últimas.

La ventaja competitiva del modelo del distrito, en cambio, se basa en la diversidad de las culturas locales que caracterizan los distritos industriales concretos, determinando la variedad de los productos específicos en ellos fabricados y vendidos con éxito en el mercado mundial, para satisfacer un amplio sector de núcleos de necesidades diferenciadas, percibidas por grupos de consumidores distribuidos por todos los rincones del mundo.

De aquí precisamente deriva la importancia del reconocimiento de la diversidad cultural y del respaldo activo a su valorización, para contrastar la globalización de las empresas transnacionales, a favor de una globalización que abarque las diferentes economías locales, sin igualarlas, y que represente además una posibilidad para las economías locales de los países en vías de desarrollo.

\section{5.- Bibliografía}

Becattini, G. (2000): Dal distretto industriale allo sviluppo locale, Torino, Bollati Boringhieri.

Corò, G. y Micelli, S. (1999): Distretti industriali e imprese trasnazionali, Sviluppo locale, vol. VI, n. 10 , p. 16-40.

EEG (1995): Enciclopedia dell'Economia, Milano, Garzanti.
Gramsci, A. (1975): Quaderni del carcere, vol, 1, Torino, Einaudi.

Hines, C. (2000): Localization. A Global Manifesto, London, Earthscan. 